\title{
Performance of SDMA Multi-User Detection Techniques for Walsh-Hadamard-Spread OFDM Schemes
}

\author{
Matthias Münster and Lajos Hanzo ${ }^{1}$ \\ Dept. of Electronics and Computer Science, \\ University of Southampton, SO17 1BJ, UK. \\ Tel: +44-1703-593 125, Fax: +44-1703-594 508 \\ Email:lh@ecs.soton.ac.uk \\ http://www-mobile.ecs.soton.ac.uk
}

\begin{abstract}
Multiple reception antenna assisted Space Division Multiple Access (SDMA) techniques designed for wireless OFDM systems have recently drawn wide interests. These techniques facilitate the implementation of effective multi-user detection algorithms at the receiver, such as Minimum Mean-Square Error (MMSE) detection, Successive Interference Cancellation (SIC), Parallel Interference Cancellation (PIC) or Maximum Likelihood (ML) detection. In this contribution we will investigate the performance of the MMSE and PIC detection techniques in the context of a Walsh-Hadamard spreading assisted SDMA OFDM system.
\end{abstract}

\section{INTRODUCTION}

Space Division Multiple Access (SDMA) concepts incorporating effective multi-user OFDM detection techniques have recently been addressed in a variety of publications. More specifically, detection techniques typically employed in CDMA systems, such as Minimum MeanSquare Error (MMSE) combining [1-3], Successive Interference Cancellation (SIC) [3-5], Parallel Interference Cancellation (PIC) [5], Maximum Likelihood (ML) detection [3,6] and Maximum Likelihood Sequence Estimation (MLSE) [5, 7] have recently been tailored to support the operation of SDMA systems. A further technique, which can be used by both single- and multi-carrier CDMA systems [8], potentially supporting multiple simultaneous users at the cost of a reduced throughput for each individual user, is spreading of the transmitted signal by means of orthogonal codes, such as for example Walsh-Hadamard sequences. In the context of single user OFDM systems, spreading has been recognized as an ef-

VCT2001 Fall Conference, October 7-11, 2001, Atlantic City, NJ The financial support of the European Union under the auspices of the Pan-European TRUST project and that of the EPSRC, Swindon UK is gratefully acknowledged

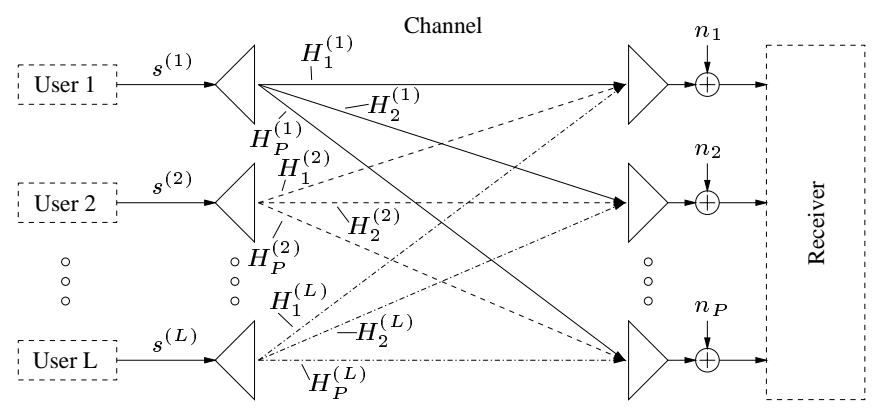

Fig. 1. Schematic of an SDMA uplink scenario, where each of the $L$ users is equipped with a single transmit antenna and the receiver is assisted by a $P$-element antenna front-end.

fective technique for exploiting the multipath diversity offered by a wideband channel. Hence, in this contribution we combine multiple reception antenna assisted SDMA concepts, where each user accesses the entire available frequency band, with subcarrier spreading [8]. Our investigations will assess the impact of spreading on the MMSE and PIC detection techniques. The outline of the paper is as follows. In Section II we introduce the SDMA signal model, while in Section III the principles of spreading are explained. In Section IV simulation results are presented for various system configurations in terms of the number of users supported. Our conclusions are offered in Section V.

\section{The SDMA SignAL Model}

In Figure 1 we have portrayed an SDMA uplink transmission scenario, where each of the $L$ simultaneous users is equipped with a single transmission antenna, while the receiver capitalizes on a $P$-element antenna front-end. The $(P \times 1)$-dimensional vector of complex signals, $\mathbf{x}[\mathbf{n}, \mathbf{k}]$, received by the $P$-element antenna array in the $k$-th subcarrier of the $n$-th OFDM symbol is constituted by the 
superposition of the independently taded signals associated with the $L$ users sharing the same space-frequency resource. The received signal was corrupted by the Gaussian noise at the array elements. The indices $[n, k]$ have been omitted for notational convenience during our forthcoming discourse, yielding:

$$
\mathbf{x}=\mathbf{H} \mathbf{s}+\mathbf{n},
$$

where the $(P \times 1)$-dimensional vector $\mathbf{x}$ of received signals, the vector $\mathbf{s}$ of transmitted signals and the array noise vector $\mathbf{n}$, respectively, are given by:

$$
\begin{aligned}
\mathbf{x} & =\left(x_{1}, x_{2}, \ldots, x_{P}\right)^{T} \\
\mathbf{s} & =\left(s^{(1)}, s^{(2)}, \ldots, s^{(L)}\right)^{T} \\
\mathbf{n} & =\left(n_{1}, n_{2}, \ldots, n_{P}\right)^{T}
\end{aligned}
$$

The frequency domain channel transfer factor matrix $\mathbf{H}$ of dimension $L \times P$ is constituted by the set of channel vectors of the $L$ users:

$$
\mathbf{H}=\left(\mathbf{H}^{(\mathbf{1})}, \mathbf{H}^{(\mathbf{2})}, \ldots, \mathbf{H}^{(\mathbf{L})}\right),
$$

each of which hosts the frequency domain channel transfer factors between the single transmitter antenna associated with a particular user $l$ and the reception array elements $p \in\{1, \ldots, P\}$ :

$$
\mathbf{H}^{(\mathbf{l})}=\left(H_{1}^{(l)}, H_{2}^{(l)}, \ldots, H_{P}^{(l)}\right)^{T},
$$

with $l \in\{1, \ldots, L\}$. Regarding the statistical properties of the components associated with the vectors in Equation 1 , we assume that the complex data signal $s^{(l)}$ transmitted by the $l$-th user has zero-mean and unit variance. The AWGN noise process $n_{p}$ at any antenna array element $p$ exhibits also zero-mean and a variance of $\sigma^{2}$. The frequency domain channel transfer factors $H_{p}^{(l)}$ of the different array elements $p \in\{1, \ldots, P\}$ or users $l \in\{1, \ldots, L\}$ are independent, stationary, complex Gaussian distributed processes with zero-mean and a different variance of $\sigma_{l}^{2}$ [1].

\section{WALSH-HADAMARD TRANSFORM SPREADING}

During the $n$-th OFDM symbol period prior to transmission the set of $K$ data symbols $s_{i}^{(l)}[n, \hat{k}], 0 \leq \dot{k} \leq K-1$ of the $l$-th user, which are associated with individual subcarriers in a scenario using no spreading, is subjected to the Walsh-Hadamard Transform (WHT) [8] in the spread scenario. As a result, each subcarrier-related symbol at the output of the transform block contains a contribution of all the original subcarrier data symbols that are to be transmitted. At the receiver after subcarrier-by-subcarrier based

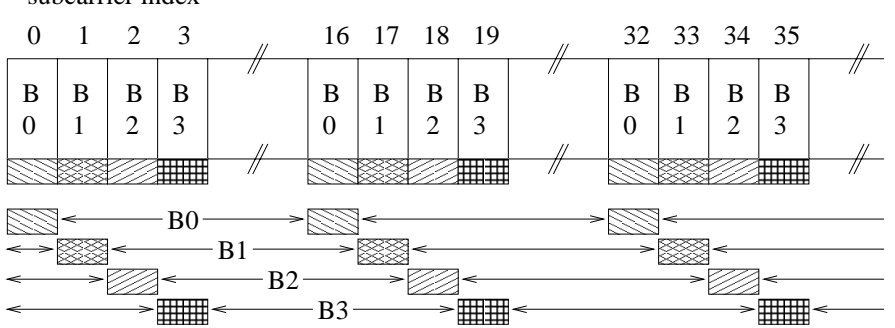

Fig. 2. Illustration of WHT subcarrier spreading using a limited WHT blocksize, which is a fraction of the total number of subcarriers hosted by the OFDM symbol.

channel equalization in the single-user single-reception antenna scenario or after array combining in the multiuser multi-reception antenna assisted SDMA scenario despreading is performed. Despreading is invoked separately for the different users in the SDMA scenario. Due to the operations of spreading and despreading combined with MMSE based frequency-domain equalization the adverse effects of low SNR subcarriers on the average BER performance is potentially improved. This is a direct consequence of spreading, since even if the signal corresponding to a specific chip is obliterated by a deep channel fade, after despreading its effects are spread over the WHT-length. Hence there is a high chance of still recovering all the partially affected subcarrier symbols without errors.

Since the employment of WHTs having a high transform length, such as for example 512, as required in case of the indoor WATM system model employed in our investigations in Section IV would impose a high computational complexity, we partition the OFDM symbol into several WHTs. This is also justified by the observation that most of the channel's diversity can be exploited with the aid of a relatively short spreading length, as illustrated in Figure 3. Furthermore, depending on the particular delay spread profile of the channel, the OFDM symbol bandwidth of $K$ subcarriers can be divided into $K / M$ interleaved blocks of size $M$ each, which are separately subjected to the WHT. More specifically, the $i$-th WHT block contains subcarriers having indices $j$ given by:

$$
j=i+r \frac{K}{M}, \quad 0 \leq r \leq M-1,
$$

where according to our definition both the first WHT block and the first OFDM subcarrier are represented by an index of zero. In Figure 2 we have further illustrated the operation of WHT based spreading applied to blocks of an identical size, where each block hosts only a fraction of the total number of $K$ subcarriers associated with the OFDM symbol. More specifically, in this particular example the OFDM symbol is composed of 16 interleaved WHT blocks and the subcarriers which are 16 frequency positions apart 


\begin{tabular}{|c|c|c|c|}
$f_{s}$ & $f_{c}$ & $N_{\text {path }}$ & $K M S(\tau)$ \\
\hline $225 \mathrm{MHz}$ & $60 \mathrm{GHz}$ & 3 & $16.9 n s$ \\
\hline \hline FFT length & cycl. prefix & $f_{d, \max }$ & $f_{d, \max }^{\prime}$ \\
\hline 512 & 64 & $2778 \mathrm{~Hz}$ & $1.235 * 10^{-5}$ \\
\hline
\end{tabular}

TABLE I

PARAMETERS OF THE INDOOR WATM ENVIRONMENT [9].

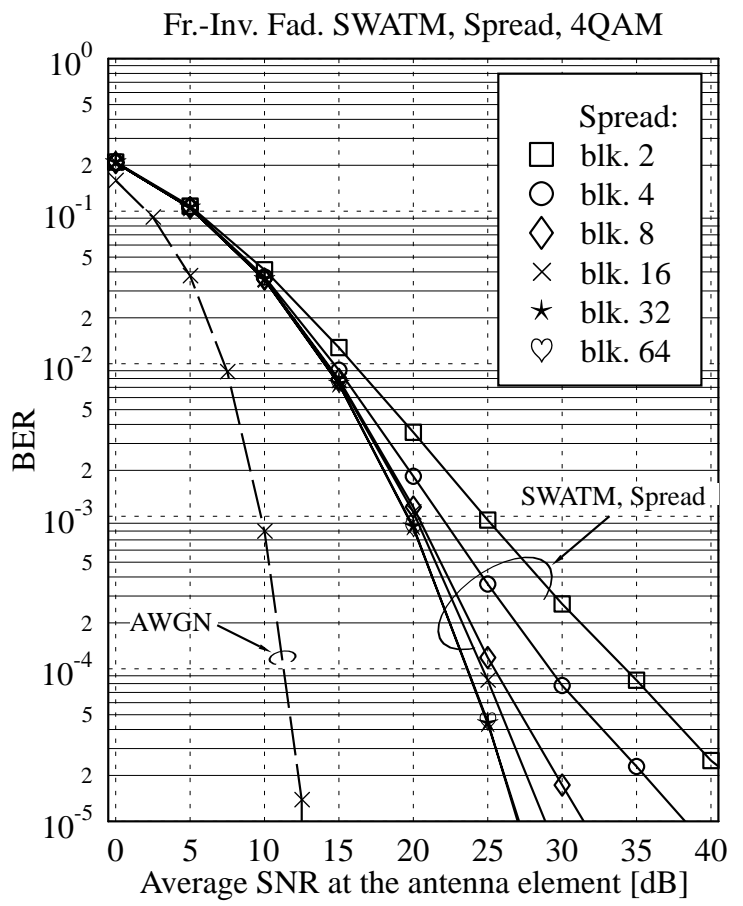

Fig. 3. BER performance of a Walsh-Hadamard spreading assisted, 4QAM modulated single reception antenna, single user OFDM system parameterised with the spreading blocksize in the context of the 'frame-invariant' fading indoor WATM channel model of Table I [9]; ideal channel transfer function knowledge was assumed; the BER performance of 4QAM signalling over an AWGN channel has been plotted as a reference.

from each other belong to the same WHT block.

The WHT spreading assisted OFDM system can be viewed in terms of the average BER achieved as a 'fully loaded' downlink Multi-Carrier Code Divison Multiple Access (MC-CDMA) scheme. As a result of the orthogonality of the WHT, the operations of MMSE based frequency-domain equalization and despreading are decoupled both in the single-user single reception antenna as well as in the multi-user multi-reception antenna scenario.

\section{Simulation Results}

Simulation results have been obtained for the indoor WATM system- and channel model of $[9,10]$. The associated parameters are listed in Table I. We commenced our
Fr.-Inv. Fad. SWATM, Adapt./Spread, 4QAM

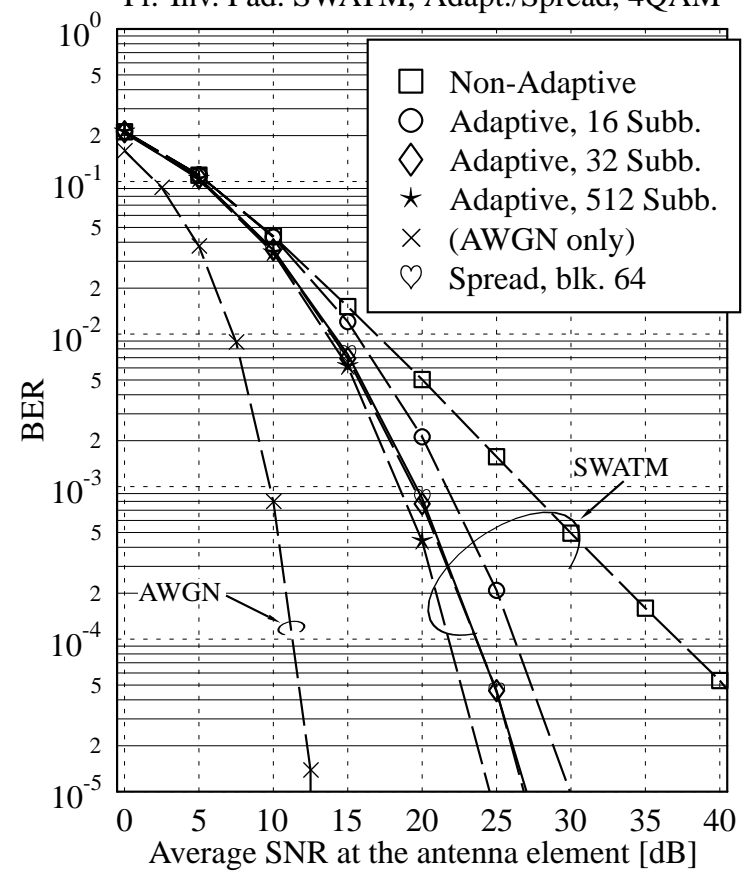

Fig. 4. BER performance comparison between a 4QAM modulated single reception antenna, single user standard OFDM system, an OFDM system employing four-mode ('no transmission', BPSK, 4QAM, 16QAM), constantthroughput, zero-delay signalling based adaptive modulation using 16 or 512 subbands, and a Walsh-Hadamard spreading assisted OFDM system employing a spreading blocksize of 64 subcarriers in the context of the 'frameinvariant' fading indoor WATM channel model of Table I [9]; ideal channel transfer function knowledge was assumed; the BER performance of 4QAM signalling over an AWGN channel has been plotted as a reference.

investigations by assessing the impact of Walsh-Hadamard spreading using different spreading code lengths on the BER performance of a 4-level Quadrature Amplitude Modulation (4QAM) single reception antenna, single user OFDM system in the indoor WATM channel environment of Table I. MMSE-based frequency domain channel equalization $[8,11]$ was performed at the receiver. The corresponding results are portrayed in Figure 3. We observe that as a consequence of the residual 'multiple-access' interference imposed by the spread signals of the different subcarriers hosted by each WHT block, the BER performance is not particularly sensitive to the WHT block length provided that it is in excess of 32 subcarriers for the SNRs of our interest. It should be noted that the benefit of spreading is directly related to the diversity potential offered by a specific dispersive channel. More specifically, the higher the channel's delay spread, the less separated are the frequency-domain fades, hence allowing a lower WHT length for achieving as high a randomization of the 


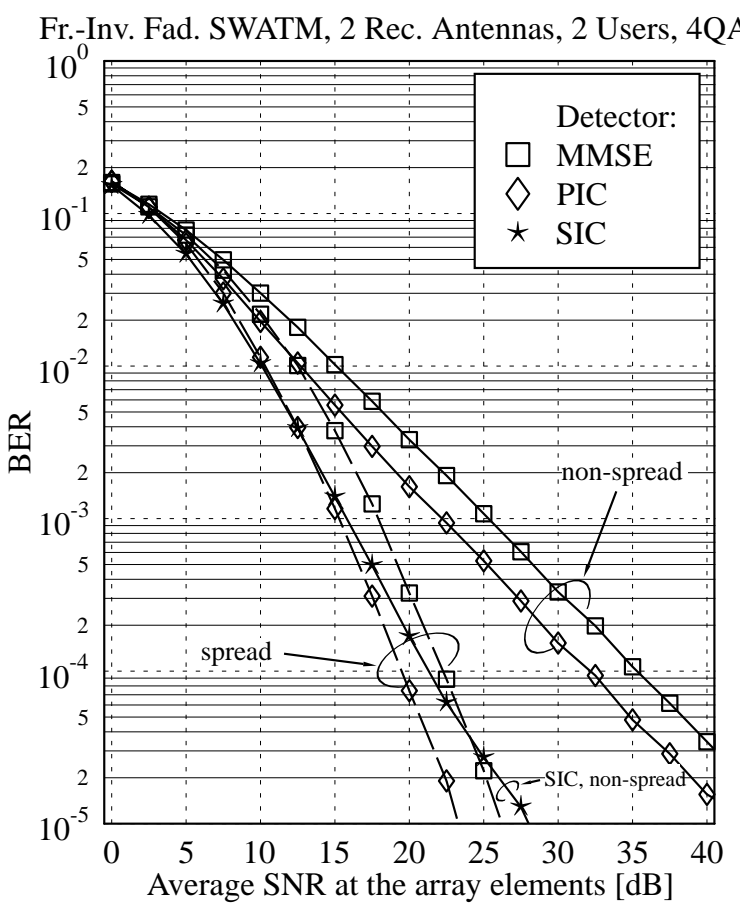

Fig. 5. BER performance comparison between MMSE, PIC or SIC multi-user detection assisted, 4QAM modulated, two reception antenna, two user OFDM systems, in the context of an SDMA channel environment as portrayed in Figure 1, where each independently fading channel is characterized by the parameters of the 'frame-invariant' fading indoor WATM channel model given in Table I [9]; the systems employing MMSE and PIC multi-user detection were further assisted by Walsh-Hadamard spreading using a blocksize of 16 subcarriers; ideal channel transfer function knowledge was assumed.

frequency-domain fading effects as possible.

In our next investigations we compared the BER performance of the WHT assisted OFDM system to a nonspread OFDM system and to an OFDM system employing adaptive modulation [9], under the constraint of having a target throughput equivalent to that of the fixed mode 4QAM modulated OFDM system. The modulation mode adaptation regime employed a total of four modes, namely 'no transmission', BPSK, 4QAM and 16QAM transmission. In order to reduce the signalling overhead required, the modulation modes were assigned on a subband basis, where each subband hosted either one or a number of subcarriers. Specifically, using one subcarrier per subband allowed us to determine the upperbound performance of the system. Furthermore, the best case scenario of perfect channel transfer function knowledge was invoked in the process of determining the optimum modulation mode assignment. The corresponding simulation results are illustrated in Figure 4. We observe that in the specific indoor WATM channel environment, assuming the separa-

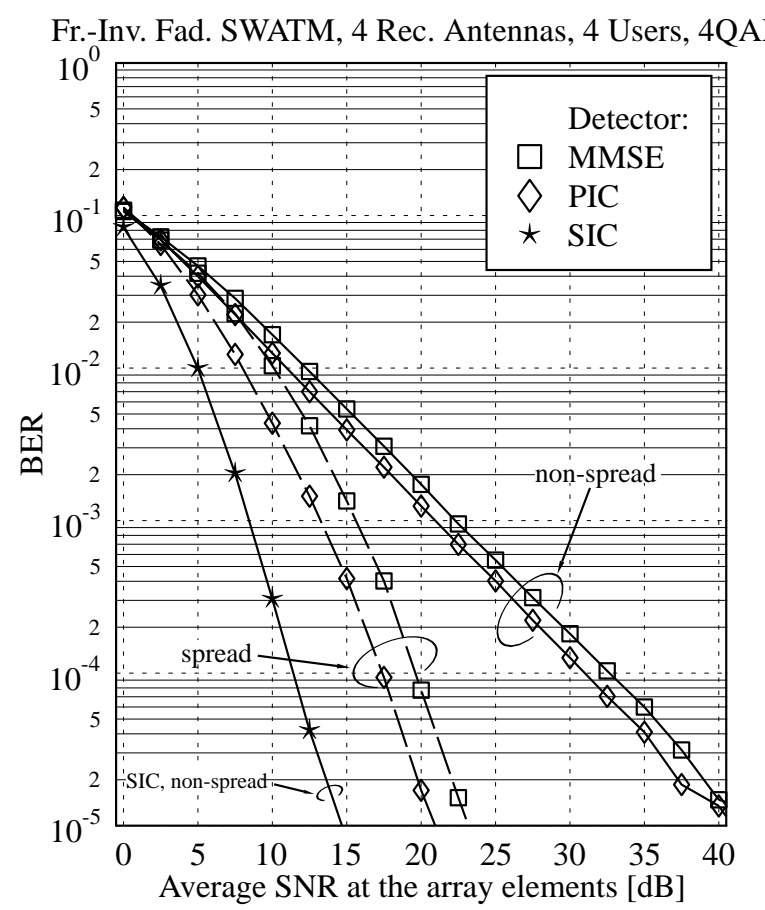

Fig. 6. BER performance comparison between MMSE, PIC or SIC multi-user detection assisted, 4QAM modulated, four reception antenna, four user OFDM systems, in the context of an SDMA channel environment as portrayed in Figure 1, where each independently fading channel is characterized by the parameters of the 'frame-invariant' fading indoor WATM channel model given in Table I [9]; the systems employing MMSE and PIC multi-user detection were further assisted by Walsh-Hadamard spreading using a blocksize of 16 subcarriers; ideal channel transfer function knowledge was assumed.

tion of the total bandwidth into 32 equal-sized subbands, each hosting 16 subcarriers, the OFDM system employing adaptive modulation exhibits the same performance as the spread OFDM system, for a WHT blocksize of 64 subcarriers. By contrast, a hypothetic system assigning an individual modulation mode to each subcarrier outperformed the WHT OFDM scheme by about $2 \mathrm{~dB}$. Hence, taking also into account the signalling overhead required by the adaptive modulation scheme, as well as its limited applicability to relatively slowly varying channels in the absence of channel transfer function prediction techniques, we conclude that subcarrier spreading is a more convenient approach to exploiting the wideband channel's diversity potential.

Our further aim was to investigate the applicability of WHT based spreading in the context of an SDMA-OFDM system, where the signals of $L$ simultaneous users each equipped with one transmission antenna are separated at the BS with the aid of a $P$-element antenna array. This scenario was portrayed in Figure 1. In our investigations 
we invoked the MIMSE [1-3] and the PIC [D] based mult1user detection approaches, while SIC [3-5] was not directly applicable to a spread OFDM system. This is because in a specific subcarrier or subband in each iteration the highest-power user is detected first, followed by subtraction of its sliced and remodulated signal from the residual composite multi-user signal received by each antenna. Since the WHT based spreading is performed across subcarriers spaced apart from each other as far as possible, for the sake of maximising the achievable diversity effect, these subcarriers would potentially require a different SIC detection order. Hence not all the symbols of a specific user contained in a WHT block are available at the same time for demodulation. Initial simulation results have been obtained for a two reception antenna, two user SDMA scenario. The results are portrayed in Figure 5. We observe that the PIC detector using WHT based spreading outperforms the SIC detector in the non-spread case, which, following the philosophy of the M-algorithm, tracked two possible symbol states from each node. By contrast, the least complex multi-user receiver, namely the MMSE detector performed about $2.5 \mathrm{~dB}$ worse, than PIC, which was also observed in the non-spread scenario. Again by contrast, in case of a four reception antenna, four user SDMA scenario characterized in Figure 6, the SIC detector applied in the non-spread scenario outperforms both spreading assisted arrangements, namely those employing MMSE and PIC based detection. Still, in comparison to an arrangement using no spreading, both the MMSE and PIC detection assisted SDMA scenarios exhibit a significant performance gain in the presence of spreading.

\section{CONCLUSIONS}

From the investigations conducted we conclude that WHT based subcarrier spreading can be effectively employed in the context of SDMA/OFDM scenarios supporting a moderate number of users in conjunction with MMSE and PIC multi-user detection techniques, in order to exploit the diversity potential offered by the wideband channel. While spreading assisted PIC detection slightly outperformed the SIC detection assisted system using no spreading in the two-reception antenna, two-user scenario, in OFDM scenarios supporting a high number of users, SIC detection - which was not directly applicable to scenarios using spreading - was demonstrated to be more effective.

\section{REFERENCES}

[1] Y. Li and N. R. Sollenberger, "Adaptive Antenna Arrays for OFDM Systems with Cochannel Interference," IEEE Transactions on Communications, vol. 47, pp. 217-229, Feb 1999.
[2] M. Münster, T. Keller, and L. Hanzo, "Co-Channel Interference Suppression Assisted Adaptive OFDM in Interference Limited Environments," in Proc. of Vehicular Technology Conference, vol. 1, (Amsterdam, Netherlands), pp. 284-288, IEEE, September 19-22 1999

[3] P. Vandenameele, L. V. der Perre, M. Engels, and H. D. Man, "A Novel Class of Uplink OFDM/SDMA Algorithms for WLAN," in Proc. of Global Telecommunications Conference - Globecom'99, vol. 1, (Rio de Janeiro, Brazil), pp. 6-10, IEEE, December 5-9 1999.

[4] G. D. Golden, G. J. Foschini, R. A. Valenzuela, and P. W. Wolniansky, "Detection Algorithms and Initial Laboratory Results using V-BLAST Space-Time Communication Architecture," IEE Electronics Letters, vol. 35, pp. 14-16, Jan. 1999.

[5] M. Münster and L. Hanzo, "Co-Channel Interference Cancellation Techniques for Antenna Array Assisted Multiuser OFDM Systems," in Proc. of 3G-'2000 Conference, vol. 1, (London, Great Britain), pp. 256-260, IEE, March 27-29 2000.

[6] G. Awater, A. v. Zelst, and R. v. Nee, "Reduced Complexity Space Division Multiplexing Receivers," in Proc. of Vehicular Technology Conference, vol. 1, (Tokyo, Japan), pp. 11-15, IEEE, May 15-18 2000

[7] M. Speth, A. Senst, and H. Meyr, "Low Complexity SpaceFrequency MLSE for Multi-User COFDM," in Proc. of Global Telecommunications Conference - Globecom'99, vol. 1, (Rio de Janeiro, Brazil), pp. 2395-2399, IEEE, December 5-9 1999.

[8] K. Fazel and G. Fettweis, Multi-carrier Spread Spectrum \& Related Topics, ISBN 0-7923-7740-0. Kluwer, 2000.

[9] L. Hanzo, W. Webb, and T. Keller, Single- and Multi-carrier Quadrature Amplitude Modulation. IEEE Press- John Wiley, April 2000.

[10] T. Keller and L. Hanzo, "Adaptive multicarrier modulation: A convenient framework for time-frequency processing in wireless communications," Proceedings of the IEEE, vol. 88, pp. 611-642, May 2000.

[11] K. Fazel and G. Fettweis, Multi-carrier Spread Spectrum, ISBN 0-7923-9973-0. Kluwer, 1997. 